\title{
On CSSCI Citation Analysis as Application Prospect of Humanities and Social Sciences Evaluation Method
}

\author{
Qian $\operatorname{Sun}^{1} \&$ Hongwei Sun ${ }^{2}$ \\ ${ }^{1}$ Department of Social Science, Northwest University for Nationalities, Lanzhou, China \\ ${ }^{2}$ School of Environmental and Municipal Engineering, Lanzhou Jiaotong University, Lanzhou, China \\ Correspondence: Qian Sun, Department of Social Science, Northwest University for Nationalities, Lanzhou 730030, \\ China. E-mail: esqian@126.com \\ Received: December 24, 2013 \\ Accepted: January 19, $2014 \quad$ Online Published: February 16, 2014 \\ doi:10.5430/jms.v5n1p108 \\ URL: http://dx.doi.org/10.5430/jms.v5n1p108
}

This work was supported by the Fundamental Research Funds for the Central Universities of Northwest University for Nationalities (Grant No.31920130129).

\begin{abstract}
CSSCI (Chinese Social Sciences Citation Index) has a certain influence and reorganization in Chinese humanities and social sciences, this paper tries to analyze the reasons for the spreading influence and characteristics of CSSCI to explore application prospect of citation analysis. It is pointed out that the CSSCI Citation analysis would be an important and feasible method of academic evaluation of humanities and social sciences. Establishing and improving CSSCI is bound to become an important aspect of constructing China's evaluation system of humanities and social sciences.
\end{abstract}

Keywords: CSSCI citation analysis, academic evaluation, quantitative evaluation, qualitative evaluation

\section{Introduction}

As a method for Chinese humanities and social science literature information query and evaluation, CSSCI not only offers a variety of information retrieval way, but also provide region, institutions, subject, scholars and various types of the cited frequency, impact factor, periodical influence scope, geographical distribution, half-life, such as statistical analysis of data, so as to provide basis for the evaluation of academic activities. The influence of CSSCI is increasingly and widely used. On the one hand, the ministry of education has made CSSCI data as an important indicator for university institutions and base evaluation, achievement awards, project establishment, famous journals evaluation, personnel training, etc. On the other hand, the personnel and scientific research system reform have been carried out in many colleges and universities, aims at combining the teacher's title post engaging with scientific research work, and formulate the scoring criteria of CSSCI in scientific research performance.

\section{The Analysis of the Causes of Growing CSSCI Influence}

The growing influence of CSSCI is decided by the needs of the reality and the characteristics of CSSCI itself.

First of all, since the nineties of the 20th century, due to domestic rampant, appraisal of scientific research projects, research achievements and awards in the peer-reviewed often become a mere formality, already made just and holy peer-reviewed lost to the position and role of the past. This comes at a time when the international journal of science citation index (SCI) and literature metrology spread and popular at home, so it was found that use of SCI and its literature measurement indicators, the scientific research workers and publications for the quantitative evaluation and management of scientific research performance, has certain objectivity and operability, and in practice shows that some unique advantages beyond the peer review at the time. In addition, SCI uses mathematical method for scientific analysis, which has fully rationality and reliability, its quantitative analysis is also adapted to the needs of the quantitative evaluation and digital management. Therefore, citation analysis and SCI is gradually favored by some people and institutions, become a "magic weapon" in the academic evaluation and scientific research management. And as inspired by SCI and developed by CSSCI, citation index certainly become a popular academic humanities and social science evaluation and management in the field of Chinese development. 
Secondly, a scientific research innovation includes the contribution of this research field, social benefits and social effects, etc. The contribution was reviewed mainly through peer experts, and the benefits and effects was determined by a certain quantitative indicators. If the range of application reflects its social benefits, referenced results may illustrate its academic influence. "The effect and influence of the humanities and social science research lies in whether the reference by others," this makes the CSSCI have a major impact on the determination of effect and impact for humanities and social science research.

Thirdly, although people have failed to fully master the essence of the citation analysis including SCI, CSSCI and so on, lots of research and application have shown that: firstly, it is a reflection of the quality of results. Secondly, there is a strong relation between peer reviews or it can be compared to peer review to some extent, that is to say, the citation analysis is an evaluation method using the combination of quantitative and qualitative for the quality of results, which is uncertain in time and space.

\section{The Controversy of whether CSSCI Citation Analysis Could Be an Academic Evaluation Method}

There is theoretical controversy about whether the CSSCI citation analysis could be an academic evaluation method. The objections includes, one of the cited frequency can be increased by the self-citation; a paper may be frequently cited due to be refuted or as a negative example; widely used research methods or original paper providing the basic data could be more frequently cited comparing with finding a new theory or an original paper with academic insight.

First of all, since self-citation is a further research on the basis of authors' own or co-authors' work, so the high frequency of self-citation shows that it is a narrow profession or a new research field has formed. The author has to publish articles very often in order to increase the cited frequency. Despite the high quality academic papers may not be published in high-quality journals, but lower quality papers must not be published in high quality academic journals. If you want to publish in a high level of journals, you must have a lot of high level early researches; otherwise it is difficult to get published for a long time. If only published in some second-tier journals but not much others' reference, instead of a large number of abnormal high frequency self-citation, which would be noticed by the peer and relevant scientific research management departments, even by some "academic misconduct organization".

Secondly, published a low level paper may produce high referenced data theoretically, but take the initiative to criticism that paper is very rare. Being cited is very rare because of criticism and negation and which may not affect the number of citations as research results measure. In fact, researchers tend to ignore those unimportant and low-quality works. If they never tired of criticizing a result, it must have some substantial contents for the result, that is to say, the result caused numerous achievements of criticism shows that this achievement is influential. Therefore, although many new theory and the important findings often criticized by people at first, the contribution of academic achievements are established lately through criticism, discussion, exchange to finally clarify the facts and eliminate misunderstanding.

Thirdly, widely used research methods or original paper that provides the basic data may be cited more frequently than finding a new theory or writing an intelligent paper. Such as in SCI papers, an article about the protein determination was cited more frequently than Einstein's article about unified field theory, but this is not certainly means that the author's contribution of this paper is more than Einstein, just show the scientists who pay attention on protein determination are more than scientists who study unified field theory. Similar situation may also occur in the field of humanities and social science, for example, the excavation report about a new archaeological discovery may be referenced by many researchers, but papers through the excavation report work which could make significant academic innovation may not be cited frequently as an archaeological report, although its academic contribution may be greater. It should be admitted that the citation analysis is a measure of scientific research activities in a sense, and a certain cited frequency of research papers do not necessarily indicate that it is completely at first-rate. In fact this is confined to a limited research field and scope, papers providing basic data in the field of humanities and social science have pioneering significance mostly.

\section{CSSCI Citation Analysis's Application Prospects as Academic Evaluation Way}

\subsection{CSSCI Citation Analysis Has Factors for Determining the Quality of Academic Achievements}

In the early sixties and seventies of the $20^{\text {th }}$ century, the American institute for scientific information (ISI) conducted an investigation for the winners' cited frequency of the Nobel Prize for physics, chemistry, and medicine in 1962 and 1963.The cited frequency extracted from ISI in 1961 to rule out the effects of cited author's reputation because of awards.

The research results show that the average cited frequency of these authors is 30 times in their fields. Each paper's cited frequency of Nobel Prize winner is 2.9 on average, and their peer cited frequency with an average of 1.57 . The 
study expanded in1977, compiled all science paper cited frequency of Nobel Prize winner in $1961 \sim 1975$ since 1950 . The papers of 162 Nobel Prize winners were cited 2877 times on average, the cumulative citation of other authors in the index were less than 50 times on average. The above results show that on the one hand, based on citation analysis, the correlation between the quality of the judgment and the Nobel committee peer-reviewed judgment is very strong. On the other hand, in all the variables those can affect the citation rates, the quality of the paper is dominant, and strength of other variables is not enough to affect the interpretation of the cited frequency's obvious difference. And in the field of humanities and social science, despite the humanities and social science has certain particularity, it cannot quantify directly on the expression of the achievements quality like natural science achievements. However, CSSCI that followed and inherited the SCI citation analysis method is also a reflection of the quality of results. For example, Chinese humanities and social science academic influence report book published by Chinese academy of social sciences research evaluation center in Nanjing University issued the statistics of academic institutions related subjects and cited frequency from 2000 to 2004, the ranking roughly reflect the strength of the current state of the corresponding subjects academic institutions, according with the overall academic and social intuitive understanding for the academic institution status in the mass.

\subsection{CSSCI Citation Analysis Has Elements of the Qualitative and Quantitative Evaluation Method}

Qualitative and quantitative evaluation all has its reasonable factors and disadvantages under certain conditions, whether CSSCI citation analysis has elements of the qualitative and quantitative evaluation method and whether could make these two methods combine basically decided by our understanding for CSSCI. Generally speaking, CSSCI citation analysis is the quantitative evaluation method or indirect evaluation, peer review is the qualitative evaluation method or direct evaluation method. In fact, CSSCI citation analysis is not only a qualitative evaluation method, but a quantitative one.

The reason is that the value of referenced achievement was read by professional staff, which is in line with the meaning of qualitative or peer review completely, actually it is the peer review. And then to calculate the number of cited times through citation analysis, this is quantitative evaluation.

If the above view was set up, understanding of CSSCI quotation analysis and its evaluation function will be further improved. In general, analysis effect of CSSCI citation analysis for the basic and macroscopic research and medium is more noticeable, yet for "micro research, such as personal academic evaluation will be treated cautiously".

\subsection{CSSCI Citation Analysis's Limitation on Academic Achievements on Micro Level}

In conclusion, CSSCI citation analysis will appear inconformity to different evaluation objects. We should clear its limitation when evaluate on different objects, especially for individual academic work performance. First of all, the citation analysis has not yet identified the importance of some scientific research work in academic. Such as cross discipline and interdisciplinary, and more to reflect the focus and interest of academic research. Secondly, citation analysis provides a kind of measure for the impact and utility of scientific research, but it doesn't explain the nature and utility of the scientific research work. These reasons could only be explored through analyzing the content of the cited material or peer expert appraisal. In the final analysis, citation analysis does not mean to replace this analysis research; it just makes this analysis more objective and acute.

\section{Conclusion}

To sum up, the CSSCI analysis citation is an important and feasible way of academic evaluation in most cases. We can say that it is for the establishment of CSSCI that humanities and social science evaluation in China get a great promotion and development. Meanwhile, years of practice and development, CSSCI has been confirmed by academic and scientific research management as a way of humanities and social science evaluation.

Professor QiuJunping of Wuhan University says "CSSCI has important scientific theory value and practical significance". Professor CaiShushan in Tsinghua University thinks: "by the development of learning and innovating, CSSCI has become the important indexes for evaluation of art humanities and social sciences with Chinese characteristics". Therefore, CSSCI has become an important brand. Establishing and improving CSSCI is bound to become an important aspect of constructing China's evaluation system of humanities and social sciences.

\section{References}

Cheng, Ping. (2009). the academic evaluation system of humanities and social sciences. Journal of Chongqing University, 4, 71-76.

Liu, Dachun. (2009). A Survey of Evaluation of Chinese Humanities and Social Sciences. Journal of Chongqing University, 1, 45-50. 
Qiu, Jun-ping. (2002, March). SSCI: Significance, Characteristics and Amelioration. Wuhan University Journal (Social Sciences), 55(2), 232-236.

Ye, JiYuan. (2010). Approaching Evaluation System in Humanities and Social Sciences. Journal of Nanjing University, 1, 51-55.

Zhu, Shao-qiang. (2007). The characteristics of humanities and social science research and impacts on research evaluation. Journal of Chongqing University (Social Science Edition). 5. 